\title{
A TestBed for Deployment of Datacenter Switches for Training Purposes
}

\author{
E. Sumanth Reddy, K. Pavan Kumar, Hamid Shahnasser \\ School of Engineering, San Francisco State University, San Francisco, USA \\ Email: hamid@sfsu.edu
}

How to cite this paper: Reddy, E.S., $\mathrm{Ku}$ mar, K.P. and Shahnasser, H. (2017) A TestBed for Deployment of Datacenter Switches for Training Purposes. Journal of Computer and Communications, 5, 129135.

https://doi.org/10.4236/jcc.2017.53014

Received: September 20, 2016

Accepted: March 10, 2017

Published: March 13, 2017

\begin{abstract}
Today's network industry needs highly qualified engineers to understand, configure, develop and upgrade switches that can build scalable high performance and ultra-low latency networks. This paper describes a new networking laboratory that has been set up to provide hands-on experience on datacenter network switches, such as ARISTA, programming and monitoring the traffic. This paper explains the networking laboratory coursework that students carry out during the course of a semester. The laboratory consists of six hands on experiments on the latest datacenter switches from Arista Networks, two programming assignments that teaches some of the protocols used at data link layer and routing protocols used at the network layer of OSI reference model. The final two laboratory experiments use the Wireshark software tool for traffic monitoring and peeking into details of some of the protocols in TCP/IP such as ARP protocol, Ethernet and so forth. The coursework details include the switch basics, switch hardware, Extensible Operating System (EOS), configuration of Link Aggregation Control Protocol, Multi-chassis Link Aggregation Protocol, Access Control Lists, and Open Short Path First version 2 on Arista switches 7050T-64 and 7048T-4S. The programming tasks cover Highlevel Data Link Control (HDLC) and Routing Algorithms.
\end{abstract}

\section{Keywords}

Arista, Data Centre switches, Link Aggregation Control Protocol, MLAG, Access Control Lists, OSPF, Wireshark

\section{Introduction}

Networking industry which is continuously changing, poses numerous challenges for the academic institutions which are trying to keep the students up to date on the new industry trends and innovations providing them enough practical experience. New, efficient labs and curriculum are needed to cope with this 
rapidly changing industry. In this paper, a laboratory that has been set up, using latest Arista Corporation datacenter network switches is explained. Switches used in such an environment require high performance. Arista switches' new features such as Extensible Operating System (EOS), Gigabit Ethernet access and deep buffer architecture enables the switches to deliver such high performance and required low latency.

The course work introduced in this paper guides us through basic configurations, and features of the datacenter switches. Features such as routing protocols and access control lists are introduced in the experiments. Moreover, link aggregation protocols of Arista devices such as Link Aggregation Control Protocol (LACP) and Multi Chassis Link Aggregation Protocol (MLAG) are also implemented as a part of the lab experiments.

Additionally, the laboratory experiments include implementation of routing algorithms, important for demonstrating how the IP addressing and routing work, as well as experiments using Wireshark [1] software tool for traffic monitoring and analysis of various protocols.

\section{Laboratory Environment}

The laboratory has sixteen personal computers, four Arista 7050T-64 and four 7048T-4S switches. It can accommodate up to sixteen students, divided into groups of four and are given different time slots to perform their lab experiment. They are required to submit a report for each lab answering questions asked in the lab experiment, which encompasses all the tasks performed. Equipment required in each lab session includes personal computers, Arista switch, DB9 to Ethernet port connector cable and LAN cables.

These labs can be carried out virtually using a network software emulator known as Graphical Network Simulator (GNS3) along with Virtualization Software (Virtual Box). After installing GNS3, an ISO image of Arista VEOS (Virtual Extendable Operating System) is installed in the Virtual Box and path of this image is provided to the GNS3.

In GNS3, Arista switch is seen graphically and could be dragged on to the workspace to create network designs. Command Line Interface of Arista switch is accessed virtually by clicking the switch icon in GNS3. Moreover, operating system of a virtual switch resembles the operating system of a real switch and performs all the functions as if you are acting on an actual switch. All the lab experiments are carried out virtually ahead of the hands-on experiments on the actual datacenter switches in the laboratory.

Switches are accessed from a host computer using Teraterm or Putty, both terminal emulator software. SSH and Telnet are used to connect to multiple switches when a single host system is used.

The routing programming assignments and the Wireshark experiments are done individually using personal devices. However, these devices must have the integrated development environment (IDE) together with the Wireshark packet-sniffer downloaded. This allows students to compile the $\mathrm{C}$ programs and to analyze each packet. 


\section{Course Overview}

The main purpose of this course is to provide hands on experience on configuring and implementing switches in the datacenter environment. The course is divided into three different sets of laboratory assignments which include Arista Networking labs, programming of routing algorithms and Wireshark experiments. First set of labs concentrate on understanding the switching concepts, configuring the switches and implementing a particular concept of networking on Arista devices. These experiments attempt to achieve these goals by configuring the switches for a particular topology.

\subsection{Lab Experiment 1: Arista Basics}

This experiment involves introduction to the switch hardware, operating system (EOS) and components used to configure the switch. Manual and Zero-touch provisioning are described in this experiment. Students also make themselves familiar with the command line interface of the Arista EOS for the following lab experiments.

\subsection{Lab Experiment 2: Link Aggregation Control Protocol (LACP)}

This lab demonstrates the LACP protocol used in the data link layer of the OSI model. Connecting multiple Ethernet ports of one switch to multiple Ethernet ports of another switch with the goal of increasing the overall throughput, bandwidth and reduce the chances of failure. Active and Passive modes are used in LACP. In Active mode, the switch sends and receives packets, while in the Passive mode it only receives packets. Considering the switches S1 and S2 in Figure 1, the following table describes the establishment of channel in various cases [2] [3].

\subsection{Lab Experiment 3 Part 1: Multi-Chassis Link Aggregation Protocol (MLAG)}

This lab illustrates the layer 3 Multi Chassis Link Aggregation (MLAG). It is the aggregation of two or more physical links on multiple switches into a single logical link. In Figure 2, a peer link is created using port-channels between the two

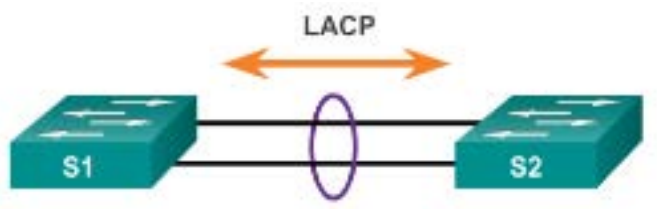

\begin{tabular}{|l|l|l|}
\hline S1 & S2 & Channel Establishment \\
\hline On & On & Yes \\
\hline Active/Passive & Active & Yes \\
\hline OniActiveiPassive & Not Configured & No \\
\hline On & Active & No \\
\hline Passive/On & Passive & No \\
\hline
\end{tabular}

Figure 1. LACP channel establishment cases [4]. 
cooperating switches known as peer switches. The primary purpose of this peer link creation is to exchange MLAG control information between the two peer switches. Peer address and MLAG domain ID are configured in both the peer switches so that each switch uses the peer address to form and maintain the peer link. MLAG domain ID configured in each switch is used to identify their peers. A virtual LAN is created between the peer switches so that they communicate with each other exclusively [3].

\subsection{Lab Experiment 3 Part 2: Multi-Chassis Link Aggregation Protocol (MLAG)}

This lab demonstrate show to connect two or more physical links on multiple switches into a single logical link known as peer link and gives the access to the peer link switches by a third switch. It is the protraction of the knowledge gained from the lab experiment 2 (LACP) and lab experiment 3 (MLA Gpart1). This lab provides an opportunity to understand Multi Chassis Link Aggregation network architecture by implementing MLAG peer link in Spine Layer and accessing peer link of spine layer from Leaf Layer using LACP implementation.

In Figure 3, the switches present in the Leaf Layer see the two peer switches present in the Spine layer as a single switch since they have the same system ID. This provides higher bandwidth links when network traffic increases, eliminates bottlenecks and provides resiliency.

In Figure 4, Switch-C is in leaf layer and peer switches (Switch-A and Switch-B) are present in the spine layer. Switch-C access the peer link using port channel 12.

By exploring the applications of the MLAG, students are exposed to data center network architecture and better understanding of it [2].

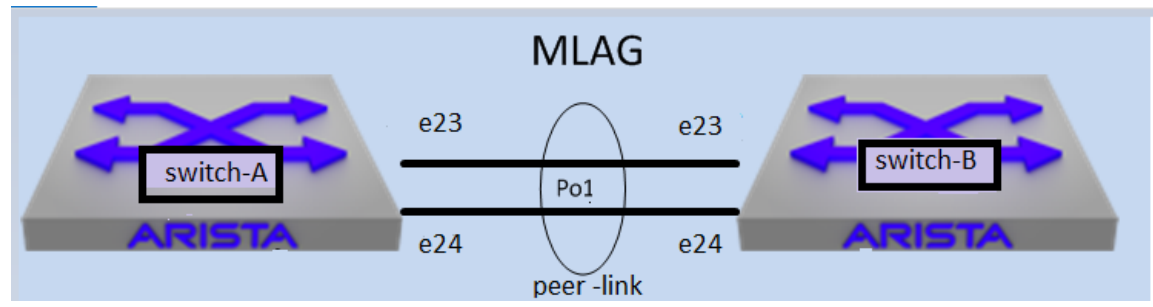

Figure 2.MLAG peer link configuration [5].

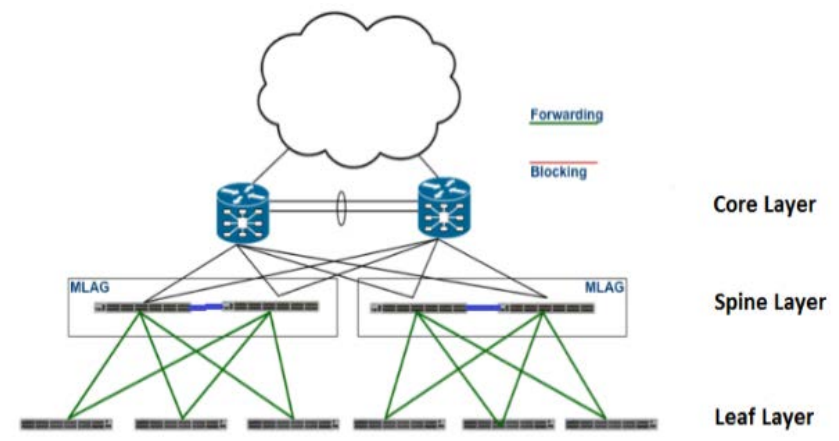

Figure 3. Leaf-spine network architecture [6]. 


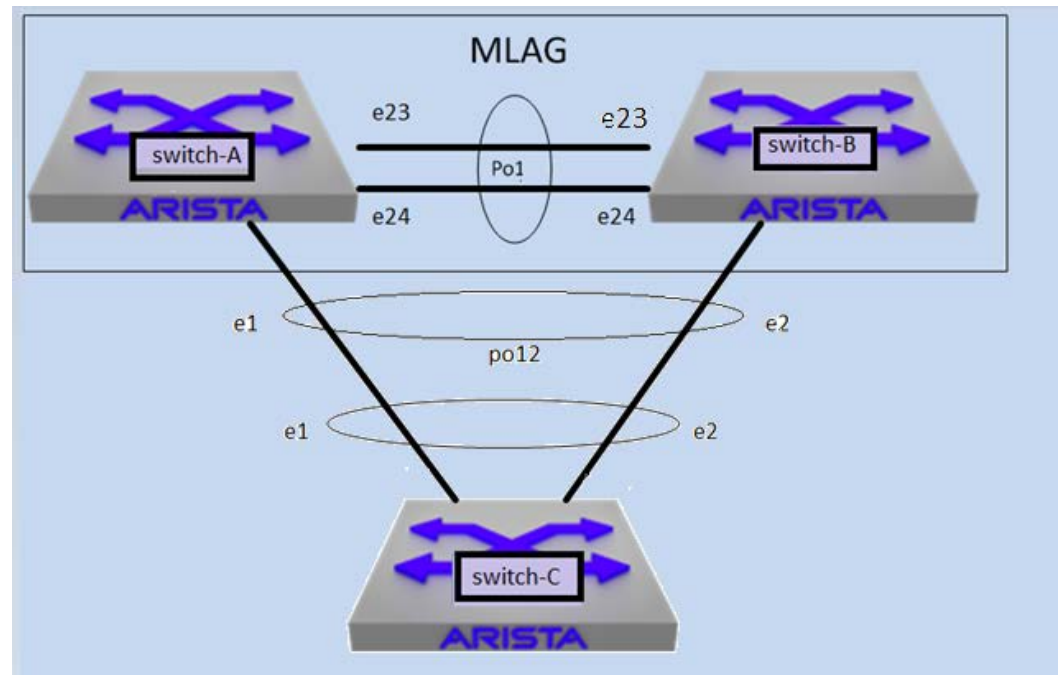

Figure 4. Multi chassis link aggregation [7].

\subsection{Lab Experiment 4: Access Control Lists (ACL)}

Access control lists are formed by a set of rules for filtering inbound flow of data packets into port channel interfaces and Ethernet ports. Arista switches in the lab support various filtering criteria such as MAC and IP addresses and TCP/UDP ports without affecting its efficiency. They are used as route maps for distribution of packets into specified domains. This experiment looks at the Access Control Lists of particular Ethernet ports or interfaces of the switches and learn to add, modify or delete them [3] [4].

\subsection{Lab Experiment 5: Open Short Path First Version 2}

The Open Shortest Path First (OSPF) [2] is a routing protocol used by IP networks. It is an interior routing protocol within a single autonomous system. This experiment explains how to configure the OSPF version 2 protocol on the Arista devices. The switch we use allows one OSPF instance. Configuration of the OSPF instance, Normal area, Stub area and Not So Stubby Area and interfaces are explained. Enabling and displaying the status of OSPF. An additional experiment on implementation of the OSPF routing algorithm is carried out in the second set of assignments in using $\mathrm{C}$ programming language.

\subsection{Lab Experiment 6: HDLC Programming Assignment}

This assignment helps students understand the Data Link Layer Protocol suite and its functionality. The $\mathrm{C}$ program students write, allows them to recognize the frames coming to this layer, by detecting the beginning and end flag delimiter of a frame. Then they can parse the frame information based on the frame field such as MAC address, data and so forth for better understanding of the protocol and writing of their own monitoring and debugging tools.

\subsection{Lab Experiment 7: Dijkstra's Routing Algorithms}

This experiment teaches the students how routing is performed in the network 
by implementing Dijkstra's [8] routing algorithm. The program written must mimic a network by providing it with a connectivity cost matrix file. Then user is prompted for a source and destination node, and the program steps through the algorithm and computes the shortest path as well as the cost of this path. This allows students to visualize and understand the routing algorithm, and how a complete end to end route can be specified by intermediate nodes, routers in this case, that have only partial connectivity information about the whole network.

The third set of experiments in this laboratory introduces Wireshark [1] software tool as a traffic monitoring and debugging tool. Experiments carried out here are accompanying experiments provided by the textbook used in this course authored by Behrouz A. Forouzan [8].

\subsection{Lab Experiment 8: Wireshark Introduction}

This assignment introduces the packet sniffer Wireshark, and explains how this software tool can extract data from any layer based on the concept of frame encapsulation. Students peer into MAC frames by answering questions about outgoing and incoming frames, the source and destination IP addresses, the total number of bytes in a frame and other relevant questions.

\subsection{Lab Experiment 9: Wireshark Address Resolution Protocol}

This lab teaches more details of Wireshark by examining the Address Resolution Protocol (ARP), which is the protocol responsible for mapping the IP addresses from layer 3 to the hardware addresses used by layer 2. Students must analyze the source and destination hardware address, the source and destination protocol address, the hardware and protocol type as well as other ARP intricacies.

\subsection{Lab Experiment 10: Wireshark \& Ethernet Protocol}

This assignment is based on the link layer protocol, and guides the student to analyze the details of an Ethernet frame. What are the fields of the frame, the data and padding that forms the payload, the destination and source addresses and other important concepts that forms this protocol.

\section{Conclusions}

A new practical laboratory has been set up to teach the important aspects of communications in a datacenter network environment.

Ten hands on experiments have been designed to provide engineers with important network knowledge that is essential to work in industry.

\section{Acknowledgements}

We would like to thank Arista Networks, for providing us with the state of the art equipment used in this laboratory. This laboratory was set up with the help of graduate students Sumanth Reddy E, Pavan Kumar K, of Electrical and Computer Engineering Embedded System program. 


\section{References}

[1] Wireshark. https://www.wireshark.org/

[2] Arista User Manual. https://www.arista.com/docs/Manuals/ConfigGuide.pdf

[3] Donahue, G.A. (2012) Arista Warrior. 1st Edition. O’Really Media, Inc., Sebastopol.

[4] Ether Channel Link Aggregation Concepts Blog. http://aymenbouzid.blogspot.com/2015/04/concepts-dagregation-de-liaison.html

[5] Configure MLAG on Arista EOS. http://www.nycnetworkers.com/real-world/configure-mlag-on-arista-eos

[6] Multi-Chassis Link Aggregation (MLAG). http://www.ipinfusion.com/blog/mlag

[7] Configure MLAG on Arista EOS. http://www.nycnetworkers.com/real-world/configure-mlag-on-arista-eos

[8] Forouzan, B.A. (2007) Data Communications and Networking. 4th Edition, McGraw Hill Higher Education, New York.

\section{Scientific Research Publishing}

Submit or recommend next manuscript to SCIRP and we will provide best service for you:

Accepting pre-submission inquiries through Email, Facebook, LinkedIn, Twitter, etc. A wide selection of journals (inclusive of 9 subjects, more than 200 journals)

Providing 24-hour high-quality service

User-friendly online submission system

Fair and swift peer-review system

Efficient typesetting and proofreading procedure

Display of the result of downloads and visits, as well as the number of cited articles Maximum dissemination of your research work

Submit your manuscript at: http://papersubmission.scirp.org/

Or contact jcc@scirp.org 